\title{
LO SCANDAGLIO DELLE DIFFERENZE. RAGION DI STATO E TIPOLOGIE DELLE FORME DI GOVERNO NELL'ITALIA DELLA CONTRORIFORMA E DELL'ETÀ BAROCCA
}

\author{
di Paolo Costantino Pissavino
}

\section{Un tema ricorrente}

Per quanto il logos tripolitikòs inserito da Erodoto nelle sue Storie (III, 80-82) avesse costituito (con la distinzione delle forme di governo in monarchia, aristocrazia e democrazia) il gesto inaugurale di una inesausta tradizione di riflessioni sulla politica, e, anzi, avesse identificato la ricerca razionale sulla politica con l'indagine su quanti - e su come - comandino nella città 1 , resta fuor di dubbio che lo sviluppo di tale tradizione nel corso dei secoli abbia conosciuto, già con Platone e Aristotele, infinite rimodulazioni e adattamenti. Invero, come notò nitidamente Norberto Bobbio, la costruzione di tipologie di forme di governo ha costituito uno dei "temi ricorrenti" nella storia del pensiero politico, indicando con tale formula quei temi "che sono stati proposti e discussi dalla maggior parte degli scrittori politici [...], e che quindi fanno parte integrante di una teoria generale della politica", anzi le varie tipologie delle forme di governo succedutesi nella storia "costituiscono uno degli aspetti attraverso cui una teoria può essere meglio caratterizzata e confrontata con altre teorie"2. Tuttavia, già a fine Ottocento, nella sua Pre-

Dipartimento di Scienze Politiche e Sociali, Università di Pavia.

1 A confermare tale indicazione valga quanto affermava N. MatTEucci, Le forme di governo, Firenze, Centro Editoriale Toscano, 2004, p. 11: "Le tipologie si costruiscono in base a dei fundamenta divisionis: per le forme di governo [...] ne possiamo indicare essenzialmente due: innanzi tutto 'chi governa' e, in secondo luogo, 'come governa'".

2 N. BobBio, La teoria delle forme di governo nella storia del pensiero politico, 
fazione al secondo volume della "Biblioteca di Scienze Politiche" da lui diretta, Attilio Brunialti (1849-1920) aveva inteso avanzare cautele, ricordando come la riflessione sulle forme di governo avesse sollevato "una disputa antica come lo Stato, insoluta come il problema della felicità umana". Aveva riconosciuto, infatti, la Difficoltà estrema dell'argomento, come intitolava un paragrafo della Prefazione, perché "Nella infinita varietà loro gli organismi politici si ribellano a una classificazione soddisfacente assai più degli organismi animati, perché gli elementi loro sono senza paragone più complessi"3. Di più, a metà del secolo XIX, uno statista e un intellettuale come George Cornewall Lewis (1806-1863) aveva testimoniato nel suo Treatise on the Methods of Observation and Reasoning in Politics una tendenziale omologazione che stava intervenendo ad annullare le differenze che per tradizione erano state riconosciute intervenire tra le forme di governo:

"The recent progress of political philosophy, however, certainly tended to shake the exclusive importance attached by the earlier speculators to political forms, and to show that some of the most important political influences pervade all civilized communities, whether under a monarchical, an aristocratic or a democratic regimen"4.

Tuttavia, una siffatta posizione non impediva a Lewis di notare, un-

Torino, Giappichelli, 1976, Premessa, p. 1. Invero, già a fine Ottocento sir Frederick Pollock aveva osservato come le forme di governo costituissero un elemento fondamentale della scienza politica: "the foundation and general constitution of the State, the forms and administration of government, and principles and methods of legislation seem naturally to fall asunder as heads under which the topics of the political science may be grouped", cfr. F. POLlock, An Introduction to the History of Science of Politics, London, Mcmillan, 1916, p. 8. La prima edizione era apparsa nel 1890. Anche per Gaetano Mosca (1858-1941) la classificazione delle forme di governo svolgeva una funzione rilevante nella costruzione della scienza sociale, che poteva effettivamente nascere allorché fossero stati individuati i "caratteri più importanti ed essenziali dei governi", cfr. G. MoscA, Teorica dei governi e governo parlamentare, in G. Mosca, Scritti politici, Volume primo, a cura di G. Sola, Torino, Utet, 1982, pp. 202-203. La prima edizione rimonta al 1884.

3 A. Brunialti, Le forme di governo. Prefazione, in G. Cornewall Lewis, E. De PaRIEU, L. PASsy, Le forme dei Governi - G.W. Hosmer, Il popolo e la politica - E. DE LAVELEY, Le forme di Governo nelle società moderne - J. STUART Mill, Il Governo rappresentativo - R. CALAMANDREI, Monarchia o Repubblica, Torino, Unione Tipografico-Editrice, 1886, p. VII, p. CVI.

4 G. C. LewIS, A Treatise on the Methods of Observation and Reasoning in Politics. In Two Volumes, London, John W. Parker and Son, 1852, vol. II, pp. 307-308. 
dici anni dopo, che "La questione delle forme di governo non ha però perduta la sua importanza", anche se le opere rivolte a descrivere "lo Stato perfetto, la migliore forma di governo [...] sono difatti quasi interamente scomparse, perocché l'opinione dominante le considera siccome inutili". Secondo Lewis, l'analisi delle forme di governo esercitava "ancora una grande influenza sullo spirito delle nazioni; essa non solo le tocca nei lor interessi, ma eccita vivamente le passioni dell'umanità e trascina moltitudini di partigiani fanatici sotto opposte bandiere"5.

Sia nella posizione espressa da Brunialti sia in quella esplicitata da Lewis emergeva, quindi, la consapevolezza della complessità che accompagnava l'istanza ineludibile per il pensiero politico di procedere alla catalogazione delle forme di governo. E tale esito ora era testimoniato dalla consapevolezza che le dinamiche storiche finivano per operare tra i vari regimi omologazioni tali da rendere più o meno surrettizie le rigide tipologie elaborate dai pensatori ${ }^{6}$, ora era confermato dal riconoscimento che l'analisi delle forme di governo non riusciva puntualmente a ricapitolare la grande varietà di assetti costituzionali che erano stati instaurati nel corso della storia. Fuor di misura che fossero le pretese tassonomiche che, autore per autore, le varie catalogazioni avevano interpretato, Lewis riconosceva anche che l'analisi delle forme di governo, a motivo dell'inevitabile confronto tra esse costruito da ciascun pensatore, si poneva come strumento di lotta ideologica e non solo come ricerca teorica.

In verità, le cautele e gli atteggiamenti critici espressi da Lewis e da Brunialti erano già ricorsi in molte scritture politiche italiane del

5 Si cita da G. C. LEwIS, Qual è la miglior forma di governo?, a cura di L. CANFORA, Palermo, Sellerio, 1996, pp. 31-32. L'opera di Lewis, pubblicata nel 1863 con il titolo $A$ Dialogue of the Best Form of Government, London, Parker Son and Bourne West Strand, già nel 1868 venne tradotta in italiano a Padova con il titolo attuale, e successivamente inclusa da Brunialti nel volume qui citato a nota 3.

6 Lewis, in una opera precedente, a tal proposito aveva affermato che "The distinction between Aristocracy and Democracy, as commonly conceived and understood, is not a logical distinction of kind, founded on a precise line of separation, but mainly a distinction of degree. [...] These two political states pass into one another by insensible degrees [... J and cannot be marked off by precise boundary", cfr. G. C. LEWIS, An Essay on the Influence of Authority in Matters of Opinion, London, W. Parker, 1849, Appendix. On the Distinction between Aristocracy and Democracy, and on Province of Political Science, pp. 405, 406, 407. 
Cinquecento e del Seicento, in cui affioravano snodi tematici che mettevano in discussione la possibilità che le pur differenti tipologie delle forme di governo finallora elaborate cogliessero effettivamente le peculiarità delle formazioni statuali così come erano andate consolidandosi. Sicché, in molti autori si riscontra un'adesione alle tipologie tramandate dalla classicità ma anche l'esaltazione della propria scelta ideologica tra principato e repubblica, insieme alla ricerca di prospettive interpretative che permettessero di descrivere più puntualmente quella "infinita varietà" che, secondo Brunialti, caratterizzava le forme di governo. Non foss'altro, quella cultura aveva ben saputo disegnare per i differenti assetti comunitativi immagini complesse, nelle quali venivano condensati i compiti plurali che l'analisi della politica aveva assunto. Esemplare, a tal proposito, resta un brano di Don Scipio di Castro (cortigiano e consigliere di principi, circa 1521-1583) che nella seconda redazione del suo trattato manoscritto intitolato Delli fondamenti dello Stato e delle parti essenziali che fondano il principe, illustrando i due "modi" in cui l'esperienza si proponeva a oggetto di indagine ("l' una è quella che ha fatto fin qui l'età del mondo [...]. L'altra è quella che fa l'uomo particolare nel corso dell'età sua"), indicava come terza parte della "esperienza universale" la "lunga narrazione" che la politica aveva saputo proporre di sé nel corso dei secoli:

"In la terza [parte] con lunga narrazione ci va mostrando tutte quelle forme di governi publici, che in diversi tempi son iti sperimentando gli Stati del mondo. E paragonandoli fra loro, va facendo giudizio del migliore. Va riconoscendo le cause delle alterazioni e ultime corruttele che hanno patito. Va dichiarando in quali contrari son iti degenerando. Va raccogliendo i remedi, con i quali possano guarirsi l'infermità di tali governi. E finalmente per la lunga esperienza di quei mali, donde si han visto nascere le sedizioni di populi, le ruine delle città, le desolazioni de' paesi, le sovversioni de' regni, le mutazioni degli Stati, va dando quelle forme di vita e di governo, quelle spezie di educazione, donde si possa sperare quel fine per il quale sono ordinate le città e le ragunanze degli uomini"’.

7 S. DI CASTRO, La politica come retorica, a cura di R. ZAPPERI, Roma, Istituto della Enciclopedia Italiana, 1978, ove a pp. 160-164 viene trascritto il capitolo Dell'esperienza tratto da Delli fondamenti dello Stato e delle parti essenziali che formano il principe; la citazione riportata nel testo si trova a pp. 161-162. Vale segnalare che nella recente edizione critica del trattato curata da Simone Testa, la lunga citazione riportata qui nel testo non 
Ordinata che fosse in questo brano la riflessione condotta da Scipio (o Scipione) di Castro (dall'indagine sui governi alla ricerca della loro "miglior forma"; dalla comprensione dei processi di degenerazione a cui andavano soggetti gli stati all'individuazione dei rimedi atti a contrastarne la rovina), pareva, a una prima lettura, seguire e ampliare a proprio modo lo schema che già Niccolò Machiavelli (1469-1527) aveva puntigliosamente descritto presiedere alla redazione del Principe nella famosissima lettera a Francesco Vettori (1474-1539) datata 10 dicembre 1513, affermando di aver "composto uno opuscolo De principatibus, dove io mi profondo quanto io posso nelle cogitationi di questo subbietto, disputando che cosa è principato, di quale spetie sono, come e' si acquistono, come e' si mantengono, perché e' si perdono"».

\section{Il ventaglio di Machiavelli}

Per il vero già nel Principe si colgono differenti tipologie delle forme politiche non sempre ricomponibili in un'identica prospettiva d'analisi.

Così, nella lettera al Vettori si può veder dispiegata una strumentazione teorica che rimandava all'aristotelismo naturalistico rinascimentale, volta a indagare: 1) la sostanza del principato (quid est?, che cosa è?) e quali fossero le specie con cui tale forma politica si presentava; 2) i modi, ovvero le tecniche, sia di conquista che di conservazione;

compare. Plausibilmente ciò è dovuto al fatto che l'edizione approntata da Testa ha seguito - come vien dichiarato nell'Introduzione - "l'intento di restituire il testo in una forma affidabile", e quindi "ha comportato la decisione di limitarsi alla trascrizione della parte della trattazione che apparve a stampa nel 1589", nel Thesoro politico, su cui vedi infra nota 51. Bisogna rilevare, tuttavia, che il trattato non è mai stato portato a termine dall'autore, e ciò può spiegare le differenti redazioni che si possiedono, e la diversità delle trascrizioni approntate da Zapperi e da Testa, ancorché quest'ultimo abbia fatto ricorso anche a estratti del codice vaticano Boncompagni D 10 su cui Zapperi aveva basato la sua trascrizione del capitolo Dell'Esperienza, cfr. S. Testa, Scipione di Castro e il suo trattato politico. Testo critico e traduzione inglese del Seicento, Manziana, Vecchiarelli, 2012, p. 14. Resta ovvia l'indicazione della ricerca condotta da Testa come più aggiornata ricostruzione dell'itinerario biografico e interpretativo della figura del di Castro.

8 N. Machiaveldi, Niccolò Machiavelli a Francesco Vettori, Firenze 10 dicembre 1513, in N. Machiaveldi, Tutte le opere, a cura di M. Martelli, Firenze, Sansoni, 1971, p. 1160 . 
infine 3) perché il principato si potesse perdere, ovvero la causa/le cause che determinasse/determinassero tale esito. Machiavelli veniva così a redigere una sorta di indice tematico che scandiva il contenuto della sua opera, dal primo capitolo (Di quante ragioni sieno e' principati e in che modo si acquistino) al ventiquattresimo (Per qual cagione li principi di Italia hanno perso li stati loro), a patto di veder rimodulata nelle tecniche di conquista e di conservazione tutta la seconda parte dell'opuscolo (capitoli XV-XXIII) relativa ai comportamenti prescritti al principe. Di più, a seguire il dettato del capitolo I, bisogna però avvertire che Machiavelli vi veniva a proporre altro e ben più ristretto sommario dell'opera, che si attagliava ai soli capitoli II-VII e che dichiaratamente descriveva nel principato una forma di stato:

"Tutti li stati, tutti e' domini, che hanno avuto e hanno imperio sopra li uomini, sono stati o republiche o principati. E' sono o ereditari, de' quali el sangue del loro signore ne sia suto lungo tempo principe, o e' sono nuovi. E' nuovi, o sono nuovi tutti, come fu Milano per Francesco Sforza, o sono come membri aggiunti allo stato ereditario del principe che li acquista, come è il regno di Napoli al re di Spagna. Sono questi domini così acquistati o consueti a vivere sotto un principe o usi a essere liberi, e acquistonsi o con le armi d'altri o con le proprie, o per fortuna o per virtù"9.

Così Machiavelli enumerava i vari tipi principato, che nella titolazione latina apposta al primo capitolo divenivano genera e non più spe$t i e^{10}$, distinguendoli secondo la titolarità del potere (principati ereditari, principati nuovi, principati misti) e i modi dell'acquisto. Tuttavia non si può non notare come, dando corso a tale distinzione, Machiavelli avesse operato per l'uno e per l'altro di tali criteri trasformazioni ed

9 N. Machiavelli, Il Principe, a cura di M. Martelli, corredo filologico a cura di N. Marcelli, Roma, Salerno, 2006, cap. I, Di quante ragioni sieno e' principati e in che modo si acquistino, pp. 63-65.

${ }_{10}$ N. MAChiaVELLI, Il Principe, cit., cap. I, Qout sint genera principatuum et in quibus modis acquirantur. Varrà ricordare che nei Discorsi sopra la prima deca di Tito Livio, per individuare le forme di governo, Machiavelli ricorreva al termine "spetie", vedi Discorsi sopra la prima deca di Tito Livio, I, 2, Di quante spezie sono le republiche e di quale fu la republica romana, cfr. N. MACHIAVELLI, Discorsi sopra la prima deca di Tito Livio, seguiti dalle "Considerazioni intorno ai Discorsi del Machiavelli" di Francesco Guicciardini, a cura di C. VIVANTI, Torino, Einaudi, 1985, p. 16. D'ora in avanti la citazione dell'opera sarà abbreviata in Discorsi. 
elisioni significative sia riguardo alla tradizione del pensiero politico sia riguardo allo stesso sviluppo argomentativo dispiegato nell'opuscolo. Vale osservare che, per quanto riguarda il principio della titolarità del potere, con la formula "principato nuovo" Machiavelli elideva dalla propria pagina ogni richiamo alla tirannide, della cui famiglia semantica infatti non si trova traccia nel Principe. Per quanto poi riguarda l'architettura argomentativa della prima parte dell'opera, nel primo capitolo Machiavelli nulla scriveva delle altre modalità di acquisto che avrebbe invece descritto nei capitoli VIII, IX-X, XI, ovvero il principato acquistato mediante scelleratezze, il principato civile che può trasformarsi in assoluto, e i principati ecclesiastici.

Vale osservare che l'opposizione operata nell'incipit del Principe fra le due forme di stato, ovvero principato e repubblica, ribadita in altri scritti in modo netto, era certo frutto di schematizzazioni e semplificazioni rispetto a una pluralità di assetti politici che Machiavelli non ignorava affatto ed era, anzi, pronto a rappresentare nella loro complessa gerarchizzazione: "ognuno sa che chi dice imperio, regno, principato, republica, chi dice huomini che comandano, cominciandosi dal primo grado et descendendo infino al padrone d'uno brigantino, dice giustizia et armi""11. Per il vero, però, proprio laddove Machiavelli pretendeva di additare solo nel "vero principato" e nella "vera republica" la compiuta stabilizzazione degli assetti possibili alla forma stato, faceva capolino nella sua scrittura una chiara instabilità semantica:

"La cagione perché Firenze ha sempre variato spesso nei suoi governi, è stata perché in quella non è stato mai né repubblica né principato che abbi avute le debite qualità sue: perché non si può chiamar principato stabile, dove le cose si fanno secondo che vuole uno, e si deliberano con il consenso di molti: né si può credere quella repubblica essere per durare, dove non si satisfà a quelli umori, a' quali non si satisfacendo, le repubbliche rovinano"12.

Per il vero, il giovane Machiavelli nelle Parole da dirle sopra la

${ }^{11}$ N. MaChiaVelli, 1512. La cagione dell'ordinanza dove la si truovi, et quel che bisogni fare. Post res perditas, in N. MACHIAVELli, Tutte le opere, a cura di M. MARTELLI, Firenze, Sansoni, 1971, pp. 37-38.

12 N. Machiavelli, Discursus florentinarum rerum post mortem iunioris Laurentii Medices, in N. Machiavelli, Tutte le opere, a cura di M. Martelli, Firenze, Sansoni, 1971, p. 24. 
provisione del danaio, facto un poco di proemio et di scusa (1503) aveva ben netta la distinzione che interviene tra la forma della comunità politica e i tipi di governo, che per altro venivano da lui ricordati secondo la tradizionale tripartizione: "Tucte le città le quali mai per alcun tempo si sono governate per principe soluto, per optimati, o per populo come si governa questa [ovvero Firenze], hanno avuto per defensione loro le forze mescolate con la prudentia, perché questa non basta sola, e quelle o non conducono le cose o, conducte, non le mantengono"13. Si metta pure da parte il precorrimento che questo brano testimonia nei confronti delle immagini della "golpe" e del "lione" contenute nel famoso capitolo XVIII del Principe, bisogna tuttavia notare che la forma monarchica di governo ricordata nelle Parole da dirle sopra la provisione del denaio è il principato assoluto, quel tipo di monarchia che nel Principe si presenta solo per due accenni che ricorrono nella parte conclusiva del capitolo IX, Del principato civile ${ }^{14}$. Al di là di questi accenni al principato assoluto, nel Principe è contenuta una vera e propria analisi delle forme con cui il principato poteva essere governato, venendo, a proprio modo, a precorrere la distinzione che, come vedremo successivamente, Jean Bodin operò tra forma dello stato e forma di governo nei suoi I sei libri dello Stato.

Infatti nel IV capitolo del suo capolavoro Machiavelli dava conto di una tipologia delle forme di governo principesco altrettanto importante e famosa della dicotomia di repubblica e principato annunciata nel primo capitolo. Scriveva infatti Machiavelli:

“e' principati de' quali si ha memoria si trovano governati in dua modi diversi: o per uno principe e tutti li altri servi, li quali come ministri per grazia e concessione sua adiutono governare quello regno; o per uno principe et baroni, li quali non per grazia del signore ma per antiquità di sangue tengano quel grado; questi tali baroni hanno stato e sudditi proprii, li quali li ricognoscono per signori e hanno in loro naturale affezione. Quelli stati che si governono per uno principe e per servi hanno il loro principe con più autorità,

${ }^{13}$ N. Machiavelli, Parole da dirle sopra la provisione del danaio, facto un poco di proemio et di scusa, N. MachiaVelli, Tutte le opere, a cura di M. MarTelli, Firenze, Sansoni, 1971, p. 11.

${ }^{14}$ N. Machiavelli, Il Principe, cit., cap. IX, Del principato civile, pp. 169-170: "Sogliono questi principati [ovvero quelli civili] periclitare, quando sono per salire dall'ordine civile all'assoluto [...] E el principe non è a tempo ne' periculi a pigliare l'autorità assoluta". 
perché tutta la sua provincia non è alcuno che riconosca per superiore se non lui; e, se obediscano alcun altro, lo fanno come ministro e offiziale di questo e non li portano particulare amore. Li essempli di queste dua diversità di governi sono ne' nostri tempi el Turco e il re di Francia"15.

Machiavelli opponeva quindi al governo dispotico esercitato dal sultano di Costantinopoli la forma di governo che caratterizzava la monarchia di Francia, segnata da un dualismo di poteri tra re e baroni presentato come irrisolto: "el re di Francia è posto in mezzo d'una multitudine antiquata de signori in quello stato, riconosciuti da' loro sudditi et amati da quelli, hanno le loro preminenzie, non le può il re tòrre loro sanza suo periculo"16. Nel capitolo XIX delineava, però, altra e differente immagine del regno di Francia, ricordandolo "Intra regni bene ordinati e governati a' tempi nostri", perché "in esso si truovano infinite constituzioni buone, donde depende la libertà e la sicurtà del re; delle quali la prima è il parlamento e la sua autorità" 17 . Infatti Machiavelli qualificava in questo modo l'azione di stabilizzazione operata appunto dal parlamento in funzione della conservazione dell'autorità regia:

“Perché quello che ordinò quello regno, conoscendo l'ambizione de' potenti e la insolenza loro, e iudicando essere loro necessario uno freno in bocca che li correggessi, e dall'altra parte conoscendo l'odio dello universale contra a' grandi fondato in sulla paura, e volendo assicurarli, non volle che questa fussi particolare cura del re, per torli quel carico che potessi avere co' grandi favorendo i populari, e co' populari favorendo e' grandi; e però costituì uno

15 N. Machiavelli, Il Principe, cit., cap. IV, Per quale cagione el regno di Dario, il quale da Alessandro fu occupato, non si ribellò da'sua successori dopo la morte di Alessandro, pp. 100-101.

16 N. Machiavelli, Il Principe, cit., cap. Iv, Per quale cagione el regno di Dario, il quale da Alessandro fu occupato, non si ribellò da'sua successori dopo la morte di Alessandro, p. 102.

${ }^{17}$ N. MACHIAVELLI, Il Principe, cit., cap. XIX, In che modo si abbia a fuggire lo essere sprezzato e odiato, p. 250. Per l'importanza che Machiavelli ascriveva ai parlamenti nel sistema politico francese si vedano anche le pagine dei Discorsi in cui esaltava il regno di Francia, perché "vive sotto le leggi e sotto gli ordini più che alcuno altro regno. Delli quali leggi ed ordini ne sono mantenitori i parlamenti, e massime quel di Parigi: le quali sono da lui rinnovate qualunque volta ei fa una esecuzione contro ad un principe di quel regno, e che ei condanna il re nell'esecuzione delle sue sentenze", cfr. N. MachiaVelli, Discorsi, III, 1, A volere che una setta o una republica viva lungamente, è necessario ritirarla spesso verso il suo principio, pp. 362-363. 
giudice terzo [il parlamento appunto, in particolare quello di Parigi] che fussi quello che senza carico del re battessi e' grandi e favorissi e' minori: né poté essere questo ordine migliore né più prudente, né che sia maggiore cagione della sicurtà del re e del regno. Di qui si può trarre un altro notabile, che li principi debbono le cose di carico fare subministrare a altri, quelle di grazia a loro medesimi. E di nuovo concludo che uno principe debbe stimare e' grandi, ma non si fare odiare dal popolo"18.

Emergeva così un'altra categorizzazione machiavelliana delle forme politiche a sottolineare la funzione fondamentale che le istituzioni, in questo caso i parlamenti, venivano a svolgere al fine di garantire e stabilizzare il sistema politico nel suo complesso, rafforzando nel contempo l'autorità del re nei confronti delle tensioni sociali da cui era attraversato il Paese. Infatti, come già nel capitolo IX, anche nel capitolo XIX l'arena politica era descritta essere segnata da "dua umori" animati da profonda conflittualità: i grandi e il popolo. Tuttavia non si può non riconoscere come il risultato di tale confronto fosse ben differente, perché differente risultava il ruolo che le forze sociali venivano ad assumervi: così, se la scena urbana descritta dal processo di formazione del principato civile vedeva dipendere il successo del principe dall'appoggio concesso dai grandi o dal popolo ${ }^{19}$, nel regno di Francia l'autorità del principe veniva ad affermarsi reprimendo la loro accesa conflittualità grazie all'azione del parlamento. Di più: se nel capitolo IX dallo scontro tra grandi e popolo erano viste sortire tre forme di governo che rimodulavano la tradizionale tipologia tripartita ${ }^{20}$, nel capitolo XIX, a

\footnotetext{
18 N. Machiavelli, Il Principe, cit., cap. XIX, pp. 250-252: In che modo si abbia a fuggire lo essere sprezzato e odiato.

${ }^{19}$ N. Machiaveldi, Il Principe, cit., cap. IX, Del principato civile, p. 163: "si ascende a questo principato o con il favore del popolo o con quello de' grandi”, p. 163.

${ }^{20}$ N. Machiavelli, Il Principe, cit., cap. IX, Del principato civile, pp. 163-164: “in ogni città si truovano dua umori diversi; e nasce da questo che il popolo desidera non essere comandato né oppresso dai grandi, e li grandi desiderano comandare e opprimere il populo; e da questi dua appetiti diversi nasce nelle città uno de' tre effetti, o principato, o libertà $o$ licenza", ossia un assetto monocratico del potere, oppure un governo repubblicano, o, ancora, un governo popolare segnato dalla corruzione e dal disprezzo delle leggi. Per l'uso del lemma "licenzioso" per descrivere la forma degenerata di governo popolare cfr. N. MachiaVelli, Discorsi, I, 2, Di quante spezie sono le republiche e di quale fu la republica romana, p. 19, ove descriveva il processo di corruzione delle forme di governo: "il principato facilmente diventa tirannico, gli ottimati con facilità diventano lo stato di pochi, il popolare senza difficultà in licenzioso si converte".
} 
connotare il regno di Francia, si affermava la formula "stati bene ordinati e governati", che con ogni evidenza andava oltre tale tipologia.

\section{Aristotele: il metodo scientifico e le differenze}

Per il vero, così come si è visto per Machiavelli, in molti autori all'affermazione di una rigida e netta classificazione delle forme politiche faceva seguito un'indagine assai più approfondita, intesa a illustrare ulteriori articolazioni della tipologia inizialmente proposta. Già lo stesso Aristotele, appellandosi nella sua Politica al "metodo scientifico"21, aveva programmaticamente dichiarato che "si deve esaminare se bisogna ammettere una sola forma di costituzione o più forme, e se più forme, quali sono, e quante e quali le differenze tra di loro"22, venendo in questo modo a sottolineare il valore euristico della "differenza".

Operando nel terzo libro della Politica la distinzione tra le forme rette (regno, aristocrazia, politia o democrazia retta) e quelle degenerate (tirannide, oligarchia, democrazia), lo Stagirita veniva a fondarla sul fatto che "Quando l'uno o i pochi o i molti governano per il bene comune, queste costituzioni necessariamente sono rette, mentre quelle che badano all'interesse di uno solo o dei pochi o della massa sono deviazioni'. $\mathrm{Ne}$ conseguiva che "Delle forme monarchiche quella che tiene d'occhio l'interesse comune siamo soliti chiamarla regno", mentre "La tirannide è una monarchia che persegue l'interesse del monarca"23. Appare quindi evidente che, nella classificazione aristotelica, monarchia (ovvero il governo di uno) è genere rispetto alle sue specie 24 : regno e tirannide. Tut-

21 Aristotele, Politica, a cura di R. Laurenti, Roma-Bari, Laterza, 1993, III, 8, 1279 b 15: "è proprio di chi in ogni investigazione segue un metodo scientifico, e non guarda solo all'aspetto pratico, non tralasciare né trascurare alcunché, bensì chiarire la verità su ogni cosa".

22 Aristotele, Politica, cit., III, 6, 1278 b 6-9. A ben comprendere il senso dell'indagine condotta da Aristotele bisogna ricordare che per lui la costituzione "è l'ordinamento delle varie magistrature d'uno stato e specialmente di quella che è sovrana suprema di tutto" (III, 6, 1278 b 9-11) e che "costituzione significa lo stesso che governo e il governo è l'autorità sovrana dello stato", cfr. ARISTOTELE, Politica, cit., III, 7, 1279 a 26-27.

${ }^{23}$ Aristotele, Politica, cit., III, 7, 1279 a 28-32.

24 Per un esempio del rapporto di subordinazione di genere, specie e individuo, cfr. Aristotele, Categorie, v, 2 a 16-19: "un certo uomo esiste nella specie uomo, e genere di questa specie è animale". 
tavia, l'analisi condotta da Aristotele non si fermava alla prima distinzione tra forme rette e forme degenerate, ma per ciascun genere di governo veniva a individuare distinte specie. Così del regno veniva a indicare cinque forme: infatti, essendosi chiesto "se c'è un solo genere di regno o abbia più forme", lo Stagirita aveva concluso che "è facile per lo meno rendersi conto che [il regno] abbraccia più generi, e che il sistema di governo non è uno in tutti". In tale prospettiva aveva appunto enumerato cinque forme: 1) "la costituzione laconica", che "si ritiene che sia un regno sostanzialmente conforme alla legge [...]. Questa forma di regno, dunque, è una specie di comando militare di capi assoluti e perpetuo"; 2) "i regni di alcune popolazioni barbariche: hanno tutti quanti un potere simile alle tirannidi, ma sono conformi alle leggi ed ereditari"; 3) gli esimneti: "si tratta, per dirla in modo semplice, di una tirannide elettiva, diversa da quella barbarica, non perché non fosse conforme alla legge, ma solo perché non era ereditaria", inoltre si esercitava su "sudditi ben disposti"; 4) le monarchie regie dei tempi eroici "spontaneamente accettate ed ereditarie, conformi alla legge"; 5) infine "si ha la quinta forma, qualora un individuo singolo sia sovrano d'ogni affare" 25 .

Come vale notare, tale ampia catalogazione veniva a qualificare per alcuni regni una chiara collocazione geografica (Sparta e i regni barbarici) e per tutti una precisa modalità di esercizio del potere: la conformità alla legge, riconosciuta anche quando il regno barbarico era definito come un "dispotismo ereditario" o l'esimnetia era presentata come una "tirannide elettiva"26. Tuttavia, successivamente Aristotele operava una sorte di sem-

25 Aristotele, Politica, cit., III, 14, 1285 a $1-1285$ b 30.

${ }^{26}$ A chiarire la contraddizione apparentemente costituita dall'ascrizione dell'esimnetia alla forma retta di governo propria al regno, vale forse ricorrere al commento che venne steso a metà del Cinquecento da Bernardo Segni (1504-1558): "Il terzo [tipo di regno] è l'Esimnete, che è quasi il Dittatore che s'usavasi in Roma: e dice di tale ch'egli è (per dir così) come una Tirannide; che vuol dire che non è Tirannide veramente". Anzi, Segni rafforzava le sue considerazioni non solo osservando che la tirannide era regime corrotto, ma anche affermando che "chi è eletto alla Signoria, giustamente non si può dir Tiranno", cfr. Trattato dei governi di Aristotele tradotto di Greco in lingua vulgare Fiorentina da Bernardo Segni gentil'huomo \& Accademico Fiorentino, In Firenze, Appresso Domenico Torrentino Stampator Ducale, 1549, 1. III, cap. X, Del Regno, p. 167. Sulla complessa figura di questo intellettuale fiorentino, storiografo ma anche volgarizzatore di Aristotele, si veda il contributo di S. BIOnda, Segni, Bernardo, in Dizionario Biografico degli Italiani, vol. XCI, Roma, Istituto della Enciclopedia Italiana, 2018, (on line). D'ora in avanti la citazione dell'opera sarà abbreviata in DBI. 
plificazione nei confronti di tale catalogo, osservando che "Più o meno sono due, per così dire, le forme di regno che si devono esaminare, questa [ovvero quella assoluta] e quella laconica: molte delle altre si trovano in mezzo tra queste, giacché in esse i re hanno sovranità minore che nella monarchia assoluta e maggiore che in quella laconica" 27 . Se tale riflessione si mostra essere sintesi esigente di una disamina fino a quel punto articolata e diffusa nell'evidenziare molteplici forme di regno, proprio per tale motivo non si può disconoscere la centralità che viene assegnata al concetto di "differenza" nella redazione dei libri III e IV della Politica, dedicati proprio all'analisi delle forme di governo. In verità lo Stagirita poneva l'accento sulla funzione euristica assolta dal concetto di differenza proprio al termine dell'analisi dei vari tipi di costituzione:

"Si è detto, quindi, per quale motivo esistano più forme di costituzione e perché ce ne sono delle altre, oltre quelle comunemente ammesse (infatti non c'è soltanto un'unica democrazia e lo stesso vale per le altre) e ancora quali sono le differenze tra loro e per quale causa succede che si diano tali differenze $[\ldots]]^{2} 28$.

Da questo esempio, inoltre, resta evidente come le tipologie delle forme di governo elaborate da Aristotele, così come dagli autori che seguirono nel tempo, proponessero successivi adattamenti dell'analisi della multiforme struttura della realtà politica al caleidoscopio prodotto dalla propria strumentazione teorica.

\section{Bodin e la critica del "labirinto senza fine" delle forme di governo}

A rimodulare a proprio modo la tipologia aristotelica, vale ricordare che Jean Bodin (1529-1596) usò il concetto di sovranità inteso come "potere assoluto e perpetuo che è proprio dello Stato"29, così

27 Aristotele, Politica, cit., III, 15, 1285 b 34-38.

28 Aristotele, Politica, cit., IV, 13, 1297 b 28-34.

${ }^{29}$ Come è noto, il capolavoro di Bodin ebbe a fine Cinquecento una traduzione italiana, I sei libri della Republica [...], tradotti di lingua Francese nell'Italiana da Lorenzo Conti gentil'huomo genovese, In Genova, Appresso Girolamo Bartoli, 1588, tuttavia per chiarezza le citazioni saranno tratte da J. Bodin, I sei libri dello Stato, Volume primo, a cura di M. Isnardi Parente, Utet, Torino 1964, 1. I, cap. VIII, Della sovranità, p. 345. 
da definire "per monarchia lo Stato in cui la sovranità è in mano di un solo principe, per democrazia quello in cui tutto il popolo partecipa ad essa, per aristocrazia quello in cui partecipa ad essa una minoranza del popolo". Balza subito evidente che Bodin veniva a considerare monarchia, aristocrazia e democrazia come assetto e architettura della comunità politica - ovvero Stato - che poteva essere differentemente governata. Con nettezza da subito scartava la distinzione tra governi "buoni e cattivi" - delineata, come si è visto, da Aristotele nella Politica, e prima ancora da Platone nel Politico ${ }^{30}-$ lamentando la "grande varietà" di forme politiche che in tal modo si sarebbe ricavata:

"Un tale criterio di distinzione ha permesso a molti di fissare più di tre forme di Stato, ma se tale opinione fosse giusta, se cioè veramente si dovesse commisurare i regimi degli Stati alla stregua delle virtù e dei vizi, ne risulterebbe un numero infinito".

Sicché, a partire da tale critica Bodin veniva a porre con estrema chiarezza i fondamenti epistemici di una teoria politica rinnovata nella sua strumentazione:

"È evidente che, per avere definizioni valide a proposito di un qualche soggetto, non ci si può arrestare ai fattori accidentali, che sono innumerevoli, ma occorre cogliere le differenze essenziali e formali; si finirebbe altrimenti col cadere in un labirinto senza fine, precludendo ogni possibilità di vera scienza".

Ecco, secondo Bodin, cosa turbava l'indagine sulla politica: il "labirinto senza fine" dei "fattori accidentali", scenario che ostava alla costruzione della "vera scienza"; di contro, infatti, si poneva la necessità di distinguere le forme di governo secondo quelle differenze "formali e sostanziali", che nella logica aristotelica distinguono le specie dal genere ${ }^{31}$. Se si fosse trascurata la via della "vera scienza", e si fosse atta-

30 "Governare secondo o contro le leggi suddivide ciascuna di esse [costituzioni]" in una forma retta e in una forma degenerata, cfr. Platone, Il Politico, 302e.

${ }^{31}$ Varrà ricordare che, nella tradizione dell'aristotelismo, la dimensione della politica non resta per nulla ignota alla costruzione della logica; si veda, ad esempio, uno dei trattati più diffusi e commentati nel corso del Medioevo, l'Isagoge di Porfirio. Proprio 
gliata l'indagine sulla dimensione multiforme dei caratteri accidentali, per Bodin si sarebbe giunti a un risultato paradossale:

"per questa via si potrebbe arrivare fino a foggiare definizioni di Stati non solo secondo il criterio delle virtù e dei vizi, ma anche in base a qualità indifferenti. Per esempio si potrebbe incominciare a distinguere il caso che il re fosse stato eletto per la sua forza da quello in cui fosse stato eletto per la sua bellezza, o per la sua nobiltà, o per la sua ricchezza, cose tutte indifferenti; [...] in tal modo si potrebbe arrivare a foggiare tanti tipi di monarchia quante sono le diverse qualità degli uomini”.

Di qui la ferma conclusione a cui giungeva Bodin al fine di consolidare la sua analisi sulla base dell'evidenza che mutamenti qualitativi producevano alterazioni che non modificavano "essenzialmente la natura delle cose":

"Poiché dunque la qualità non modifica la natura delle cose, affermeremo che non ci sono che tre regimi o tre forme di Stato: la monarchia, l'aristocrazia, la democrazia"32.

Tuttavia era stato lo stesso Bodin, rifiutando la bimillenaria tradizione del governo misto $^{33}$, a dilatare le maglie strette della sua tipologia tripartita delle forme di stato, e a rivelare quello che definiva "un segreto di Stato che non è mai stato trattato da nessuno" 34 . Infatti per lui "Regime e governo sono cose nettamente differenti":

"il regime può essere monarchico ma con governo democratico, se il principe permette a tutti di partecipare alle assemblee degli stati, alle magistrature, agli

nell'incipit del capitolo intitolato La specie si può leggere infatti che: "La specie si dice della forma di ogni realtà, secondo quel che è stato detto: "In primo luogo una forma degna della tirannide"". Come è noto, in prima istanza, per Porfirio "la specie è ciò che è subordinato al genere", cfr. Porfirio, Isagoge, Prefazione, introduzione, traduzione e apparti a cura di G. GIRGENTI. Testo greco a fronte. Versione latina di Severino Boezio in appendice, Milano, Rusconi, 1995, p. 65.

32 J. Bodin, I sei libri dello Stato, cit., 1. II, cap. I, Di tutte le forme di Stato, in generale; e se ve ne siano solamente tre o di più, pp. 543-544.

33 Per la critica al governo misto cfr. J. Bodin, I sei libri dello Stato, cit., 1. II, cap. I, pp. 544-566.

34 J. Bodin, I sei libri dello Stato, cit., 1. II, cap. II, Della monarchia dispotica, p. 570. 
uffici, alle ricompense, senza riguardo alla nobiltà, alle ricchezze o al merito. Può essere sempre monarchico ma a governo aristocratico se il principe non conferisce potere o benefici che ai nobili o ai più meritevoli o ai più ricchi”.

E tale peculiare distinzione e integrazione tra forma di stato e forma di governo ovviamente veniva a dilatare la tipologia tripartita di forme di stato delineata inizialmente dallo stesso Bodin, che, per parte sua, non perdeva occasione per sottolineare l'errore in cui era incorso la precedente cultura politica: "Questa varietà di forme di governo ha tratto in errore alcuni portandoli a porre forme miste di Stato; senza rendersi conto che il governo di uno Stato è ben altra cosa dalla sua amministrazione e dal modo di governarlo"35. Per il vero, sarebbe ora fuor di misura seguire il lunghissimo attacco condotto da Bodin contro i sostenitori del governo misto. Piuttosto, vale notare il fatto che Bodin veniva a dilatare ulteriormente la sua analisi delle forme di governo introducendo un'ulteriore tipologia che, stavolta, distingueva tre diverse forme di monarchia secondo la titolarità dell'esercizio del potere: "ogni monarchia è regia, dispotica o tirannica. Non si tratta di tre diversi regimi, ma solo di un modo diverso di esercitare il governo in uno stato a regime monarchico". Dopo tale affermazione, Bodin procedeva immediatamente a chiarire in che consistessero le differenze che correvano tra le tre diverse forme di monarchia: "Dunque la monarchia regia o legittima è quella in cui i sudditi ubbidiscono alle leggi del re e il re alle leggi di natura, restando ai sudditi la libertà naturale e la proprietà dei loro beni". La monarchia dispotica, invece, era la forma di governo che il vincitore di una guerra instaurava sul popolo sconfitto: "La monarchia dispotica è quella in cui il principe si è fatto signore dei beni e delle persone stesse dei sudditi per diritto d'armi e guerra giusta, e governa i sudditi come un capo di famiglia i suoi schiavi". Infine, la monarchia tirannica "è quella in cui il monarca calpesta le leggi di natura, abusa dei liberi come di schiavi, dispone dei beni dei sudditi come beni propri"36.

Tuttavia, tale tripartizione creava problemi sia di tipo epistemologico

35 J. Bodin, I sei libri dello Stato, cit., 1. II, cap. II, Della monarchia dispotica, p. 570.

36 Vale aggiungere che siffatta tripartizione viene a replicarsi anche nelle altre forme semplici di governo: "Le stesse differenze si riscontrano nell'ambito della democrazia e dell'aristocrazia: l'una e l'altra possono essere infatti legittima, dispotica o tirannica nel modo che ho detto", cfr. J. BodIn, I sei libri dello Stato, cit., 1. II, cap. II, Della monarchia dispotica, p. 570. 
sia di tipo più squisitamente politico, dei quali si mostrava ben consapevole lo stesso Bodin, pronto a sottolineare come la realtà storica fosse assai complessa e non compiutamente riconducibile entro rigidi schemi classificatori. Infatti riconosceva che: "può darsi il caso che uno stesso principe sia monarca dispotico di una parte dei suoi sudditi, re di un'altra parte di essi, tiranno dei rimanenti. Può darsi che tratti da tiranno i ricchi e nobili, rivolgendo tutto il suo favore al popolo minuto" 37 . Di più, appare evidente - e questo resta il problema più squisitamente politico - come Bodin venisse in realtà a distinguere per la monarchia una forma retta e due degenerate, perché sia il monarca dispotico che quello tirannico calpestavano le leggi di natura, che erano poste a fondamentale baluardo della libertà e della proprietà dei sudditi ${ }^{38}$. Di tale deriva Bodin era ben conscio, tanto da premurarsi di rispondere alle possibili osservazioni in questo modo:

"se è vero in certa misura che fare degli uomini liberi degli schiavi e impadronirsi di ciò ch'è di proprietà altrui è contro la legge di natura, è anche vero che, per consenso comune di tutti i popoli, ciò ch'è stato conquistato con guerra legittima passa in proprietà del vincitore e i vinti divengono suoi schiavi, cosicché non si può dire che una signoria conquistata in tal modo equivalga senz'altro alla tirannide" 39 .

${ }^{37}$ A riprova della complessità che la realtà effettuale poteva mostrare, Bodin aggiungeva con realismo che: "come non vi è alcun principe che non abbia qualche difetto notevole per buono che sia, così anche fra i tiranni non se ne trova nessuno, che per crudele che sia, non abbia anche qualche virtù o qualche tratto lodevole", cfr. J. Bodin, I sei libri dello Stato, cit., 1. II, cap. IV, Della monarchia tirannica, p. 592-593.

38 A confronto vale ricordare come per Aristotele i regni barbarici e l'esimnetia, che pur venivano avvicinati alla tirannide, erano, come si è visto "governi conformi alla legge".

39 J. Bodin, I sei libri dello Stato, cit., 1. II, cap. II, Della monarchia dispotica, p. 578. Varrà ricordare che in Italia assai netta fu la risposta critica nei confronti di Bodin: Fabio Albergati (1538-1606), proprio sulla divisione delle forme della monarchia, ribadiva come "i governi non dal numero, ma dalla bontà, e dal bene, o mal reggere, prendano le differenze loro" e sottolineava con forza le contraddizioni che emergevano dalle pagine dei Sei libri dello Stato: "poscia che questo politico havendo già detto, che nel formar le specie delle republiche non si guarda a buono, o cattivo, ora dice il contrario, e fa differenza la monarchia reale dalla tirannica", cfr. F. AlBERGATI, Discorsi politici. Ne i quali viene riprovata la dottrina politica di Gio. Bodino e difesa quella di Aristotele, In Roma, per Giacomo Dragondelli, 1664, Secondo libro, cap. I, Della forma delle Republiche male intesa dal Bodino, pp. 169 e 172, e Secondo libro, cap. III, Della Monarchia mal intesa, pp. 180181. La prima edizione era apparsa "In Roma, appresso Luigi Zannetti" nel 1602. Sulla figura di questo teorico della ragion di stato, per una prima indicazione, ancora utile è la voce redatta da E. FASANO GuARINI, Albergati, Fabio, in DBI, vol. I, 1960, pp. 617-619. 
Eppure, a guardare oltre le complesse indagini proposte da Bodin, bisogna osservare che, per quanto proponessero descrizioni anche minuziose dei differenti assetti di potere, non sempre le tipologie approntate dalle scritture politiche riuscivano a cogliere le specificità delle realtà statuali loro contemporanee. Vediamo perché, prendendo le mosse da una importante osservazione che Platone aveva posto nel suo ultimo dialogo, le Leggi.

\section{Costituzioni reali e paradigmi ideali}

Per il vero, soprattutto i governi misti suscitavano non poche difficoltà nell'interpretarne correttamente gli assetti, che - come aveva sottolineato Platone nelle Leggi - inducevano, in chi li osservava, giudizi contrastanti, frutto di volta in volta delle differenti prospettive e sensibilità ideologiche assunte dall'osservatore. Posizione, questa, che restava ben testimoniata, appunto nell'ultimo dialogo dal personaggio di Megillo di Sparta:

"Ebbene se io penso a quello che è la costituzione spartana, non ti so dire così con qual nome bisognerebbe chiamarla. Assomiglia alla tirannide, mi pare - infatti il potere degli efori è straordinario come sia divenuto in essa tirannico - e qualche volta invece mi pare uno stato più di tutti gli altri stati somigliante alla democrazia. D'altra parte, dire che non è un'aristocrazia è del tutto assurdo e inoltre c'è la monarchia a vita in essa e, secondo quanto si dice da tutti i popoli e da noi stessi, è la più antica di tutte."

Così Megillo - lungi dal fornire una risposta su qual mai fosse l'ottima costituzione (la monarchica, l'aristocratica o la democratica ${ }^{40}$ ), richiesta avanzata dall'Ospite Ateniese, personaggio che nel dialogo dà voce a Platone - veniva piuttosto ad ammettere la sua incapacità di definire la costituzione di Sparta: "E ora io non so, in verità, come dissi,

40 Varrà a questo proposito ricordare che nel sesto degli Assiomi politici premessi alla traduzione Delle Leggi dialogo quarto di Dardi Bembo veniva sottolineata con forza una prospettiva relativistica in merito alle forme di governo, cfr. Platone, Opere tradotte da Dardi Bembo Gentiluomo veneziano cogli argomenti e note del Serano, Volume terzo, In Venezia, al Secolo delle Lettere, presso Giuseppe Bettinelli, 1743, p. 85: "Molte sono le forme di governo opportune secondo la varietà dei luoghi”. 
rispondere all'improvvisa tua domanda, così com'è, distinguendo a quale di queste forme appartiene la nostra costituzione"41.

Nella medesima difficoltà, va detto, veniva a trovarsi anche l'altro interlocutore del dialogo, Clinia di Creta: pure Clinia, infatti, non sapeva indicare quale costituzione fosse vigente a Cnosso, difficoltà che trovava la propria ragione nel fatto che la costituzione sotto cui viveva, così come quella di Sparta, fossero costituzioni reali, risultato, cioè, di differenti assetti di potere che si erano determinati nel corso della storia, e non fossero, invece, un modello ideale: "voi miei cari - così infatti osservava l'Ospite Ateniese - siete partecipi di costituzioni reali; quelle invece che abbiamo ora nominato non sono vere costituzioni ma strutture politiche dove una parte comanda e l'altra serve e ciascuna prende il nome dalla potenza di chi domina"42. Come appare evidente, le difficoltà che, secondo Platone, si incontravano nel definire con correttezza le varie tipologie dei sistemi politici scaturivano non solo dalla complessità del disegno istituzionale che caratterizzava i governi misti ma dal fatto che le costituzioni prese in esame erano, appunto, non tipi ideali ma costituzioni reali, non costruzioni astratte ma sedimentazioni peculiari di conflitti politici e sociali.

Per il vero, alcune scritture civili italiane dei secoli XVI e XVII avrebbero cercato di superare tali difficoltà, attagliando sia il differente statuto sociale - posseduto dai cittadini - sia i meccanismi istituzionali che si erano consolidati nella storia - a raffigurazioni razionalistiche, per così dire geometrizzanti, come quella dispiegata per Venezia da Giason Denores (circa 1530-1590):

"In somma, tutto questo corpo [...] è a somiglianza di una figura piramidale. Il Gran Consiglio è come la base e il firmamento in cui si appoggiano tutti gli altri ordini. Il Principe è come la punta. Il Senato, il Collegio, il Consiglio dei Diece è come il mezzo. La cima è suprema, ma è tenue. Il firmamento è più grande, ma è infimo. Il mezzo riceve il suo stabilimento dalla sede più bassa, ma le è superiore. In quantità è maggiore al grado supremo, ma in maestà è minore" 43 .

41 Platone, Leggi, iv, 712d-712e.

42 Platone, Leggi, iv, 712e-713a.

${ }^{43}$ G. Denores, Panegirico in laude della Serenissima Republica di Venezia, Padova, P. Meietti, 1590, c. 36v. Sulla figura e l'opera di Denores, professore di filosofia morale all'università di Padova, importante resta il contributo di G. PATRIZI, Denores, Giason, in DBI, 
Né, per il vero era mancato chi, come il cardinal Guido Bentivoglio (1577-1644), avesse saputo attagliare alla nuova costruzione statuale della Repubblica delle Province Unite la silhouette della "costituzione mista" al fine di descrivere, in un quadro di ordinato organicismo, una "Republica sì potente per terra e per mare; fondata in un governo di forma sì diversa da tutte le altre":

"Cospirano dunque insieme concordemente il Prencipe, \& i popoli in questa maniera: il Prencipe contentandosi d'una autorità non del tutto assoluta; \& i popoli di una libertà moderata, in quella guisa apunto, che suol procedere il governo tra il capo, e le altre membra nel regno del corpo humano" 44 .

\section{Monarchia e repubblica: lo schermo dell'ideologia}

Tuttavia, proprio nel corso dei secoli XVI e XVII, a rendere incerto il giudizio relativo alle forme di governo analizzate, sarebbero intervenuti altri e ben differenti ostacoli, quali, soprattutto, il pregiudizio ideologico ostentato dai sostenitori della monarchia, pronti, per parte loro, a negare razionalità alla machinery istituzionale propria ai regimi repubblicani, divenendo, insomma, figurazione compiuta di quei "fanatici partigiani sotto opposte bandiere" di cui, abbiamo visto, avrebbe scritto Lewis nel suo dialogo a mezzo dell'Ottocento.

Così, affatto "informe" risultava la descrizione della forma repub-

vol. XXXVIII, 1990, pp. 768-773. Già Donato Giannotti (1492-1573), nel suo Libro della Republica Fiorentina, aveva fatto ricorso a siffatta immagine, articolando in modo quadripartito il progetto che aveva voluto delineare per le istituzioni cittadine: "Sarà adunque la nostra republica composta di quattro membri principali, del Consiglio Grande, del Senato, del Collegio e del Principe; li quali faranno un corpo piramidato la base del quale sarà il Consiglio Grande, la punta il Principe, e tra 'l Principe e il Consiglio sarà il Senato sopra il Consiglio, e sopra il Senato il Collegio", se ne segue la lezione pubblicata in A. BAIOCCHI (a cura), Storici e politici fiorentini del Cinquecento, testi a cura di S. Albonico, Milano-Napoli, Ricciardi, 1994, 1. III, cap. IV, Che la republica sarà composta di tre membri principali, p. 84. Sulla figura e l'opera di Giannotti, oltre alla ricca presentazione offertane da Albonico nel volume sopra citato, si veda S. MARConI, Giannotti, Donato, in DBI, vol. LIII, 2000, pp. 527-537.

${ }^{44}$ G. Bentivoglio, Relatione delle Province Unite di Fiandra, in G. Bentivoglio, Relationi, In Colonia, 1646 [in realtà Leida, "Impresso per Bonaventure e Abraham Elsevier, 1646”], p. 8. Sul cardinal Bentivoglio, nel 1607 nunzio pontificio a Bruxelles col titolo di vescovo di Rodi, una prima informazione si ha in A. Merola, Bentivoglio, Guido, in DBI, vol. VIII, 1966, pp. 634-638. 
blicana che Gregorio Leti (1630-1701) avrebbe saputo minuziosamente ricapitolare con un organicismo, per così dire, "decomposto" nei suoi Dialoghi Politici:

"io stimo che, nelle Republiche, tutti son sudditi, e niuno Prencipe, perché il Senato, o Consiglio, forma un corpo, ch'è tutto membra, e pure non ha forma di corpo. Ho errato, \& è meglio dire, che il Senato nella Republica è una parte di tante membra recise, che forma un corpo senza membra"45.

Così, se non informi, il corpo della repubblica e i meccanismi decisionali che ne organizzavano la vita apparivano almeno complessi e farraginosi a chi era avvezzo a relazionarsi con il principato. Infatti, a fine Cinquecento, Giacomo Mancini, agente mediceo a Genova, aveva voluto testimoniare la propria "ostinata fatica" a descrivere funzioni e finalità delle magistrature dello stato ligure, dal momento che, come sottolineava, "gli huomini stessi d'un Magistrato non sanno bene le cose di quello, non che d'una Republica intiera" 46 .

45 G. Leti, Dialoghi Politici, o vero la Politica che usano in questi tempi, i Prencipi e le Republiche italiane, per conservare i loro Stati e Signorie [...]. Prima parte, In Roma, Per Francesco Moneta, 1666, pp. 204-205, ove, a portavoce delle più diffuse opposizioni al regime repubblicano, non a caso Leti presentava il personaggio del "Consigliere" del principe. Sul poligrafo Leti, una delle figure più significative del libertinismo italiano, si veda E. BufACCHI, Leti, Gregorio, in DBI, vol. LXIV, 2005, pp. 717-723.

${ }^{46}$ Così si legge nei Discorsi sopra le forze di Genova. Capitolo Primo redatti da Giacomo Mancini nel 1598, cfr. Discorsi di Giacomo Mancini sopra le forze di Genova, e nell'ultimo sua lettera et altri Discorsi mandati al Sig. ${ }^{r}$ Agostino Spinola della Rossa, conservati presso l'Archivio di Stato di Genova, ms. 955. Non stupisca, a tale proposito, che Ansaldo Cebà (1565-1623), uno dei maggiori scrittori politici genovesi della prima metà del Seicento, avesse sostenuto che il cittadino di repubblica dovesse possedere anche una notevole conoscenza delle istituzioni e della legislazione che reggevano il suo stato. Infatti "sarà mestier, c'habbia cognitione non solamente delle leggi, che contengono la forma della republica, ma di tutte l'altre, che provveggono alle cose particolari; ch'intenda il fine, perché ciascuna fu fatta; che noti se fra esse è sconvenevolezza, o contradittione; che sappia le qualità, e l'auttorità de Magistrati; che cerchi le cagioni, perché furono istituiti; che conosca la virtù, e la sufficienza de' cittadini; c'habbia contezza della fede e del valor de' ministri; e, brievemente, che non vi sia, né costitutione, né usanza, né particolarità nella republica, onde non habbia almeno tant' informatione, che possa consigliare, e provvedere a i bisogni d'essa senza commettere i difetti, che si sogliono, per non haverne notitia", cfr. A. CEBÀ, Il Cittadino di Republica alla valorosa gioventù genovese, In Genova, Appresso Giuseppe Pavoni, 1617, p. 24. Sulla figura e l'opera di Cebà una prima indicazione si trova in C. MutinI, Cebà, Ansaldo, in DBI, vol. XXIII,1979, pp. 184-187. 


\section{Le ragioni dell'omologazione e il loro superamento}

Eppure la nettezza con cui il confronto tra monarchia e repubblica palesemente aveva strutturato la tipologia bipartita delle forme di governo trovava per altre prospettive riflessioni contrastanti. Infatti vi era chi aveva ritenuto tale tradizionale opposizione tra monarchia e repubblica un che di surrettizio, osservando come pressoché tutte le forme di governo potessero essere ricapitolate in un sol tipo. Infatti, Francesco Vettori, amico di Machiavelli, fu pronto ad avanzare questa amara notazione:

"parlando delle cose di questo mondo sanza rispetto e secondo il vero, dico che chi facesse una di quelle republiche scritte e imaginate da Platone, e, come scrive Tomma Moro inghilese essere stata trovata in Utopia, forse quelle si potrebbono dire non essere governi tirannici; ma tutte quelle republiche e principi de' quali io ho cognizione per istoria, e che io ho veduti, mi pare che sentino di tirannide" 47 .

Sicché lo scrutinio operato sulla realtà effettuale aveva portato Vettori nelle sue Istorie dall'an. MDXI insino all'an. MDXXVII a comprimere la tipologia dicotomica "principato/repubblica", descritta da Machiavelli nell'incipit del Principe, nel riconoscimento della pervasività omologante di un'unica forma di governo, dolorosamente derogatoria: la tirannide.

Di più, all'interno della cultura repubblicana si era palesata anche altra prospettiva, tesa piuttosto a notare come le differenti silhoutte di governo che avevano caratterizzato la storia delle città dell'Italia centro-settentrionale avessero conosciuto un omologante approdo elitistico. A conferma, basta leggere quanto scriveva in una sua lettera, datata 21 gennaio 1531, il letterato senese Claudio Tolomei (1492-1556):

${ }^{47} \mathrm{~F}$. VETTORI, Istorie dall'an. MDXI insino all'an. MDXXVII, in A. BAIOCCHI (a cura), Storici e politici fiorentini del Cinquecento, cit., p. 466. Sulla figura e l'opera del Vettori, oltre alla accurata presentazione redatta da Albonico per l'edizione qui sopra ricordata, si veda M. SimonetTA, Vettori, Francesco, in DBI, vol. XCIX, 2020 (on line). Vale tuttavia ricordare che anche Machiavelli considerava tutte le forme di governo, rette o degenerate che fossero, "pestifere": "Dico adunque che tutti i detti modi sono pestiferi, per la brevità della vita che è ne' tre buoni e per la malignità che è ne' tre rei", cfr. N. MACHIAVELLI, $D i$ scorsi, I, 2, Di quante spezie sono le republiche e di quale fu la republica romana, p. 24. 
"in ogni Republica, benché larga, in ogni stato, benché popolare, rare volte vi è che più di cinquanta cittadini salgano a gradi del comandare in un tempo medesimo. Né anticamente in Atene, o in Roma, né al presente o in Venezia o in Lucca sono molti cittadini che governino lo stato, benché si reggano queste terre sotto nome di republica" 48 .

Ma non è escluso, anzi, che Tolomei avesse ripreso tale intuizione dalla lettura di un brano dei Discorsi sopra la prima deca di Tito Livio in cui Machiavelli osservava che: "in tutte le repubbliche, in qualunque modo ordinate, ai gradi del comandare non aggiungono mai quaranta o cinquanta cittadini" 49 .

Tuttavia, accanto all'orizzonte di omologazioni che il pensiero politico cinque e seicentesco aveva segnalato rispetto alle tradizionali tipologie, si possono riconoscere indagini che intendevano sortire dalla genericità delle aduse catalogazioni dei governi secondo il numero degli occupanti la soglia di dominio per porre attenzione alle differenze che intercorrevano tra stati sottoposti alla medesima forma di governo. Vediamo con quali strumenti di indagine.

\section{Lo scandaglio delle differenze}

Già la Somma delle opere che ha da mandar in luce l'Academia veneta, pubblicata nel 1558 dall'Accademia della Fama voluta dal patrizio veneziano Federico Badoer (1519-1593), mostrava nella sezione dedicata alle opere di politica la stretta relazione che intercorreva tra elaborazione

${ }^{48} \mathrm{C}$. Tolomer, De le lettere lib. sette. Con una breve dichiaratione infine di tutto l'ordine de l'ortografia di questa opera, In Vinegia, Appresso Gabriel Giolito de Ferrari, 1547, Lettera a M. Gabriello Cesano, "Di Cuna a li XxI di Gennaio MDXXxI", c. 145 r-v. Sulla figura di Tolomei, cfr. F. LuCIOLI, Tolomei, Claudio, in DBI, vol. XCVI, 2019 (on line). A riconferma dell'assetto oligarchico-famigliare delle repubbliche patrizie italiane nella prima età moderna valga, ad esempio, leggere quanto scriveva della Repubblica di Genova Don Pio Rossi, cfr. P. Rossi, Convito Morale. Per gli Etici, Economici, Politici [...] Ordinato et intrecciato si della ragion di Stato, come delle principali materie militari, In Venetia, Appresso i Gueriglij, 1639, p. 107: "A Genova tutta la Republica è amministrata da quelli, che sono nati da vinti otto famiglie, \& ad altri che non sono di questa adunanza, che chiamano aggregatione, non viene dato carico alcuno".

${ }^{49}$ N. MACHIAVELl, Discorsi, I, 16, Uno popolo uso a vivere sotto uno principe, se per qualche accidente diventa libero, con difficoltà mantiene la libertà, pp. 87-88. 
della ragion di stato e la descrizione delle istituzioni e delle forze dei singoli stati. Infatti gli accademici si proponevano di pubblicare

"Cinque libri di ragion di stato, ne' quali con meraviglioso ordine si contengono tutte quelle cose, che sono necessarie, e che ragionevolmente si possono sapere, dintorno alle persone, alle corti, a paesi, et a gli habitanti, et alle forze de' Principi, sì Christiani, come infedeli, delle rep[ubliche] et d'ogni altro governo" 50 .

In tale prospettiva, quindi, la ragion di Stato si poneva come selettore di un sapere geopolitico che, ad esempio, avrebbe trovato significativa testimonianza nell'indagine condotta da Giovanni Botero (1544-1617) nella seconda parte delle sue Relazioni universali ${ }^{51}$ così come avrebbe conosciuto la propria fortuna nelle edizioni che il Thesoro politico conobbe tra fine Cinque e primi anni del Seicento ${ }^{52}$. Si veniva

50 Per il Sommario e la citazione riportata nel testo, cfr. S. TESTA, Italian Academies and Their Networks 1525-1700. From Local to Global, Houndmills, Basingstoke - New York, Palgrave Macmillan, 2015, pp. 99 sgg..

${ }^{51}$ G. Botero, La seconda parte delle Relationi Universali [...]. Nella quale si discorre della potenza de' maggior Prencipi, che siano al mondo: \& le cagioni della grandezza, e sicurezza de'loro stati, In Brescia, Appresso la Compagnia Bresciana, 1599. Nel 2015, presso l'editore Aragno di Torino, sono apparse le Relazioni Universali, a cura di Blythe Alice Raviola, in due volumi. Nella seconda parte delle Relationi universali Botero presentava la potenza degli stati descrivendone "ricchezze, governo, forze, prencipi confinanti": rielaborava in tal modo un metodo che era seguito dagli ambasciatori di Venezia nelle loro relazioni al Senato, ed era iterato nelle edizioni del Thesoro politico (cfr. infra nota 52). Vale notare che siffatto interesse geopolitico che veniva ad animare la cultura cinque e seicentesca, per il vero, aveva avuto un precorrimento nell'opera di F. SANSOVINO (1521-1586), Del governo de Regni e delle Republiche antiche e moderne [...] libri XXI, ne quali si contengono diversi ordini, magistrati, leggi, costumi, historie, \& cose notabili, che sono utili \& necessarie ad ogni humo civile e di stato. Con nuova aggiunta di più Republiche \& Regni in diverse parti del Mondo, In Venetia, Appresso gli heredi di Marchio Sessa, 1567, e conobbe nella prima metà del Seicento l'impresa della pubblicazione delle cosiddette "Repubbliche elzeviriane", pubblicate tra il 1625 e il 1649: i testi in formato tascabile in $24^{\circ}$ offrivano una sorta di enciclopedia delle alternative politiche e istituzionali che la storia mondiale offriva, dalla Grecia antica al Gran Moghol, dai principati italiani alla Respublica Hebraeorum, alla Moscovia, alla Cina. Su tale raccolta cfr. M. RossetTI, Le "repubbliche" elzeviriane nelle raccolte della Biblioteca Nazionale di Firenze. Catalogo, in "Il pensiero politico", XXVII, (1994), pp. 73-93.

52 Come è noto, la prima edizione del Thesoro politico cioè Relationi Istruttioni Trattati discorsi Varii d'Ambasciatori Pertinenti alla cognitione, \& alla intelligenza delli Stati, interessi, \& dipendenze de più gran principi del Mondo. Nuovamente impresso a benefficio 
costituendo così - sulla scorta di "relazioni", "orazioni", "discorsi”, "avvertimenti" - un ricchissimo data base di "notizie" 53 su governi e stati che, a volte integrandosi con la strumentazione teorica propria dell'aristotelismo, costituiva ulteriore modalità di indagine in una dichiarata prospettiva empirica, volta ad approfondire la conoscenza delle forme politiche che si stavano consolidando in età moderna.

Di più, la stessa ragion di stato sarebbe stata intesa da Lodovico Zuccolo come indagine efficace per saggiare le "ultime differenze" che venivano a distinguere governi che pur appartenevano alla stessa specie.

\section{Lodovico Zuccolo, la ragion di stato e le "ultime differenze"}

Invero, esemplare sarebbe stata l'analisi condotta da Zuccolo (1568-1630) nell'oracolo XI delle sue Considerationi Politiche e Morali, intitolato appunto Della ragion di stato. Vediamo perché.

In primo luogo elaborava la differenza che secondo lui veniva a separare la politica dalla ragion di stato:

"Ora con grande agevolezza potremo a pieno conoscere che differenza sia tra la politica e la ragione di stato. Abbraccia la politica [...] tutto il corpo della republica, e in conseguenza ha l'occhio al bene publico ed al privato, valendosi in parte delle leggi come sue ministre, in parte adoperando ella stessa per con-

di chi si diletta intendere, \& pertinentemente discorrere li negotii di Stato, fu stampata "Nell'Accademia Italiana di Colonia", 1589. In verità fu solo in una edizione successiva che sul frontespizio apparve la locuzione "ragion di stato": La prima [-seconda] parte del Thesoro politico in cui si contengono relationi, instruttioni, trattati, \& varij discorsi, pertinenti alla perfetta intelligenza della ragion di Stato et all'intiera cognitione de gli interessi, \& dipendenze de' più gran prencipi, \& signori del Mondo, In Milano, Appresso Girolamo Bordone, e compagni, 1600. Sul Thesoro politico, cfr. S. TESTA, Italian Academies and Their Networks 1525-1700. From Local to Global, cit., pp. 109-122.

${ }^{53}$ Non va certo dimenticata l'importanza che la parola "notizia" assunse nella letteratura politica e diplomatica tra Cinque e Seicento, importanza che viene testimoniata dall'incipit della Ragion di Stato di Giovanni Botero, testo che inaugurò, con la sua pubblicazione avvenuta nel 1589, la fiorente trattatistica sulla ragion di stato: "Stato è un dominio fermo sopra i popoli, e ragione di Stato è notizia de mezi atti a fondare, conservare e ampliare un dominio così fatto", cfr. G. Botero, Della ragion di Stato, a cura di P. BenEDETtini e R. DesCendRE. Introduzione di R. DESCENDRE, Torino, Einaudi, 2016, p. 11. Sulla biografia intellettuale di Botero, oltre alle indicazioni contenute nell'edizione qui sopra citata, ancora importante resta L. FIRPO, Botero, Giovanni, in DBI, vol. XIII, 1971, pp. 352-362. 
seguir l'intento suo. Ma la ragion di stato non s'intromette se non in quei mezzi e modi, i quali si aspettano all'introdurre e al conservare forme particolari di republiche.

In secondo luogo - ed è quel che resta più importante nello sviluppo della sua argomentazione - Zuccolo veniva conseguentemente a ritrovare nella ragion di stato uno strumento per decomporre le tradizionali tipologie delle forme di governo fino al riconoscimento di quella "spezie precisa e individua [corsivo mio] di republica che altri vuole introdurre e conservare". Vediamo come.

"E per levare ogni ambiguità, la quale potesse nascere nelle parole, dico che la ragione di stato non considera quello che assolutamente convenga alla republica, né quello del pari si appartenga alla tirannide e al regno, o pure alla oligarchia e aristocrazia, ma si travaglia intorno a quelle ultime differenze [corsivo mio], per le quali formalmente si distingue l'una spezie di governo dall'altra; né pure, verbigrazia, considera come la costituzione regia sia differente dalla tirannica o l'aristocratica dall'oligarchica, ma anco più precisamente come la forma regia di Francia sia diversa dalla regia di Spagna o la popolare svizzera dalla olandese. Né perché le medesime regole di reggimento si adattino talora a più specie di governo, tuttavia poco le risguarda, se non quanto servono a quella spezie precisa e individua [corsivo mio] di republica che altri vuole introdurre e conservare" 54 .

La lunga argomentazione dispiegata da Lodovico Zuccolo veniva scandita con estrema nettezza da una logica disgiuntiva ${ }^{55}$, tutta volta a richiamare una prospettiva dichiaratamente empirica per connotare ap-

${ }^{54}$ L. Zuccolo, Della ragione di stato, in B. Croce, S. Caramella (a cura), Politici e moralisti del Seicento. Strada-Zuccolo - Settala-Accetto - Brignole Sale-Malvezzi, Bari, Laterza, 1930, pp. 31-32. Come è noto, Benedetto Croce e Santino Caramella avevano riprodotto nella antologia da loro curata l'oracolo XI, Tyrannus suam, rex subditorum utilitatem spectat. Della ragion di Stato, presente in L. Zuccolo, Considerationi Politiche e Morali su Cento oracoli di Illustri Personaggi antichi [...], In Venetia, Appresso Marco Ginami, 1621, alle pp. 54-73. Sulla figura di Zuccolo, uno dei maggiori scrittori politici italiani del Seicento, si veda, oltre a P. C. PIssavino, Le ragioni della Repubblica. La "Città felice" di Lodovico Zuccolo, Centro Sammarinese di Studi Storici - AIEP Editore, San Marino 2007, V. LaVenia, Zuccolo, Ludovico, in DBI, vol. C, 2020 (on line). Per il concetto aristotelico di "differenza ultima" si veda soprattutto ARISTOTELE, Metafisica, VII, 12, 1307 b 8-1308 a 35: "l'ultima differenza è la sostanza della cosa, e ne costituisce la definizione".

${ }^{55}$ L. Zuccolo, Della ragione di stato, cit., p. 32: "Però, quando si dice che la ragione 
punto la "specie precisa e individua di governo". Non soltanto la proposta teorica avanzata dall'autore defletteva dalla posizione tenuta da Giovanni Botero nella sua Della ragion di Stato a sostegno di una ragion di stato squisitamente monocratica ${ }^{56}$, ma sapeva procedere a una indagine alla fine attagliata alla singolarità delle forme di governo che l'esperienza storica permetteva di cogliere. Quel che resta importante sottolineare è che in Zuccolo la ragion di stato non veniva solamente a connotare l'insieme delle prassi ("i mezzi e i modi") atte "all'introdurre e al conservare forme particolari di republiche", ma diventava grimaldello euristico per distinguere nettamente le forme di governo che caratterizzavano i singoli stati. Soprattutto, di fronte a questo brano, vale avanzare alcune riflessioni che ne illustrano anche il contesto, per nulla affatto irrilevante, e per più ragioni. In primo luogo appare evidente che se la prima parte del brano fa riferimento alle forme di governo che vengono scandite dalle tradizionali tipologie tripartite (tirannide, aristocrazia ecc.), la seconda pare rifarsi alla dicotomia, di ascendenza machiavelliana, regno/repubblica. Confronto, questo, che era per nulla sopito se si tien conto del fatto che l'opera di Zuccolo venne pubblicata nel 1621, anno in cui venne a scadere la faticosa tregua dodicennale ratificata tra Spagna e la repubblica delle Province Unite.

Sicché, una strumentazione intellettuale e pratica-ovvero la ragion di stato - che per suo statuto omologava tutte le forme di governo entro la prescrizione della conservazione, in realtà per Zuccolo permetteva di rimodulare le tipologie tripartite elaborate sin dall'antichità (monarchia, aristocrazia, democrazia) in una prospettiva ben più ampia, che rendeva ciascuna comunità politica storicamente realizzatasi un unicum irriducibile ad altre realtà. Così si apprende che per Zuccolo le differenze non erano solo quelle specifiche, ovvero quelle che distinguono, appunto, la forma tirannica dalla regia, ma anche e soprattutto quelle che intervenivano a distinguere, plausibilmente attraverso caratteristiche storiche e geopolitiche, la monarchia di Francia da quella di Spagna.

di stato mira alla introduzione e conservazione della forma di qualsivoglia specie di republica, si debbono pigliar quelle parole in un tal sentimento disgiuntivo, che meglio s'intende da quello che pur ora abbiamo detto, che forse non farebbe se più a lungo con altri termini si dichiarasse, per aver la lingua nostra strettezza di voci accomodate alla espressione di simili concetti, ad ispiegare i quali si mostra ancora fanciulla".

56 "La ragion di Stato suppone il prencipe e lo Stato (quello quasi come artefice, questo come materia)", cfr. G. Botero, Della ragion di Stato, cit., p. 11. 
Anzi, proprio in questa prospettiva Zuccolo veniva a distaccarsi da un topos interpretativo presente nei commenti cinquecenteschi alla Politica, allorché cercavano, per così dire, di attualizzare, le forme regie legittime descritte da Aristotele. Sicché, se per illustrare la "forma laconica" dai commentatori veniva richiamato il dogado veneziano, se per la forma barbarica obbligato era il rimando al "Gran Turco" ovvero l'imperatore ottomano, e per la "cosiddetta esimnetia" veniva portata a confronto la dittatura dei romani, per la quarta forma di monarchia regia venivano scelte altre immagini: infatti le monarchie "dei tempi eroici" erano descritte da Felice Figliucci (1518-1595) come "Simili ai Regni di Spagna e di Francia"57.

La qualificazione operata da Zuccolo delle ultime differenze figurava, in questo modo, l'esito di un puntiglioso esercizio di analisi empirica, attorno al quale non di rado la cultura della Controriforma era venuta a impegnarsi: "Disse uno argutamente che un non so che guastava molte cose del mondo; et non so che molte cose acconciava. Volendo dire quelle ultime differenze, quasi inesplicabili, erano quelle ancora, che davano quell'ultimo et perfetto compimento alle cose et gle lo toglieva". Così, ad esempio, scriveva il cardinal Federico Borromeo (1564-1631) nel suo Liber inscriptus argumenta ${ }^{58}$.

57 F. Figliucci, De la Politica, overo Scienza Civile secondo la Dottrina d'Aristotile. Libri otto [...] scritti in forma di Dialogo. Libro non solo utilissimo ma necessario sapere il modo, \& l'arte de' Governi de' Popoli, Regni, \& Stati, In Venetia, Presso Gio. Battista Somascho, 1583, 1. III, cap. X, Del Re, \& delle molte sorti di Re, cc. 107v-109, la citazione si legge a c. 109. Sulla figura di Figliucci e sulla sua attività di traduttore e commentatore di Aristotele, si veda D. Busolini, Figliucci, Felice, in DBI, vol. XLVII, Roma, 1997, pp. 558-560. In verità, Figliucci non faceva che ripetere quello che Bernardo Segni aveva affermato nel suo commento al medesimo capitolo. In particolare, tuttavia, Segni aveva introdotto una velata cautela in merito al paragone modernizzante, cautela che in Figliucci non sarebbe più apparsa: "La quarta specie è quella che forse è simile al Re di Franza, o di Spagna: dove tai Re sono antiquati, e hannovi havuto principio per beneficitii fatti a quei Popoli o per qualche altra honesta cagione", cfr. Trattato dei governi di Aristotele tradotto di Greco in lingua vulgare Fiorentina da Bernardo Segni gentil'huomo \& Accademico Fiorentino, cit., 1. III, cap. X, Del Regno, p. 167.

${ }^{58} \mathrm{~F}$. BORROMEO, Liber inscriptus argumenta, manoscritto conservato nella Biblioteca Ambrosiana di Milano, A 77 suss. Il Liber, con il titolo di Miscellanea adnotationum variarum apposto da diversa mano, è stato pubblicato a cura del Gruppo editoriale Zaccaria, in Milano, Biblioteca Comunale, 1985; l'argumentum citato nel testo figura al $\mathrm{n}^{\circ} 281$ e si legge a p. 114. Sul Borromeo ancora importante resta P. Prodi, Borromeo, Federico, in DBI, vol. XIII, 1971, pp. 33-42. Non si può, tuttavia , tralasciare di ricordare il rinnovamento operato negli studi federiciani dalla rivista "Studia Borromaica", anche per quanto riguarda il pensiero politico del Cardinale. 


\section{Tra Francia e Spagna: le "ultime differenze"}

A suffragare la riflessione di Zuccolo rivolta a cogliere le "ultime differenze" che intercorrevano "tra la forma regia di Francia" e quella di Spagna si possono ritrovare in Federico Borromeo alcuni interessanti confronti. Il Cardinale osservava che "i francesi deliberano assai dallo stato delle cose presenti" 59 , ma alla fine erano destinati a essere interlocutori abbastanza ingenui nella conduzione delle trattative diplomatiche:

"mi disse Cosmo segretario del duca di Parma che egli servò questo stile nel trattar con franzesi nell'ultima volta che entrò in Francia. Di fargli buono tutti i partiti, se bene impossibili che proponevano, o mal intesi. Esso medesimo si essibiva prontissimo ad ogni cosa. Con un poco di tempo poi gli andava scoprendo l'impossibilità delle cose, o vero la poca raggione che vi havevano dentro. Così si levavano d'intorno. Egli medesimi si accorgevano dello errore. In buonissimo modo, per essere essi impetuosi" 60.

Di tutt'altra attitudine apparivano dotati gli spagnoli, almeno agli occhi del Borromeo che ne descriveva attentamente le strategie diplomatiche, ora con una brevissima annotazione - "Gli spagnuoli più si curano delle circostanze de negotij che dell'essenza" - ora con argomentazioni più articolate e complesse, a segnalare elisioni e dissimulazioni. Così, se illuminante restava l'osservazione che "la corte di Spagna [sempre] non dice mai quello che dispiace et non fa mai quello che piace. Significando che non offendono in parole, né danno negative", la riflessione che seguiva illustrava puntualmente le forme della comunicazione che andavano usate con gli spagnoli per ottenerne audizione:

- Persuadere - Con gli spagnoli non basta dire una ragione sola per persuadere et convincere, ma molte bisogna amassare insieme, et far numero; et dalla moltitudine più restano persuasi, che dal valore, et una di esse ferisce che sarà la più debole. Essi temono più del silentio che delle parole ${ }^{61}$ : perché non sono molto penetrativi. Et si crucciano quando non si vuole parlare se

${ }^{59}$ F. BORromeo, Miscellanea adnotationum variarum, cit., $\mathrm{n}^{\circ}$ 271, p. 112.

${ }^{60} \mathrm{~F}$. Borromeo, Miscellanea adnotationum variarum, cit., $\mathrm{n}^{\circ} 182$, pp. 83-84.

${ }^{61}$ Differente posizione veniva espressa dalla Adnotatio no 189 : "Un cavaliere fu interrogato quello che gli pareva comparativo della corte di Spagna et di Roma. Rispose in corte di Spagna si fa professione di tacere et non so se si sappia tacere. In corte di Roma 
non a mezzo. Stimano la sobrietà nel parlare: et disprezzano quando credono di intendere il tutto.

L'analisi condotta dal Borromeo andava poi a toccare le regole di governo seguite dagli spagnoli, che venivano a preannunciare le istanze relativistiche qualificate dal brano di Zuccolo prima citato:

Stimano più le usanze loro, et il $\mathrm{mo}<\mathrm{do}>$ del lor governo, et questo vogliono seguire che la ragione di stato, et le vere universali ragioni politiche. Ciò nasce dal dubbio soverchio di errare. Stimano a maraviglia gl'ossequi et le humiliationi; perché lo ricevono per tributo, et per confirmatione della loro monarchia, nella quale più si gloriano di quello che non dimostrano 62 .

\section{La ragion di Stato, la "cognitione de'particolari”, e la politica "insegnata da filosofi"}

Invero, per Borromeo gli spagnoli intendevano seguire "il modo del lor governo" anziché la "ragion di stato" e le "vere universali ragioni politiche" che gli paiono, l'una e le altre, collocarsi su un piano di universalità dal quale si distingueva appunto "il modo del lor governo", formula che indubitabilmente veniva a esplicitare ciò che Zuccolo avrebbe qualificato tra le "ultime differenze".

Per Zuccolo, insomma, la monarchia o la repubblica avevano la loro manifestazione in individue forme di governo: la monarchia di Spagna piuttosto che quella di Francia non erano certo degli enti di ragione invarianti, ma si differenziavano nella realtà storica a motivo della modalità in cui vi si erano venute dispiegando quelle tecniche di conservazione del potere che la ragion di stato ben sapeva esplicitare.

Che così fosse, è approdo che trova una significativa conferma in un'opera di un gentiluomo genovese, Giovanni Costa, ovvero il Ragionamento sopra la triegua dei Paesi bassi pubblicato nel 1610. Illustrando infatti la ragion di stato seguita dagli spagnoli, Costa osservava che

si fa professione di parlare; et si sa parlare", cfr. F. Borromeo, Miscellanea adnotationum variarum, cit., pp. 86-87.

${ }^{62} \mathrm{~F}$. BorromeO, Miscellanea adnotationum variarum, cit., $\mathrm{n}^{\circ} 332$, p. 126. 
"Il Re Catholico, e gli Arciduchi ottimamente intendono, come si reggono, e si mantengono gli Stati; e che quella, che volgarmente si dice, \& è da pochi ben intesa ragion di Stato; non è altro che una regola giusta, $\&$ atta a governare Stati secondo la lor forma, con piena notitia dello stabilimento, natura e circostantie di essi, e de' mezzi utili, anzi necessarij, così a conservargli come ampliarli per publico loro beneficio"63.

Grazie all'uso della ragion di stato come strumento euristico, Costa, e con lui Zuccolo, "sdoganavano", per dir così, quella ricerca della "specie precisa ed individua", che - abbiamo visto - Bodin con forza rifiutava ma che rappresentava il piano pratico in cui si dispiegava la ragion di stato. Insomma, a ben guardare, con forza Zuccolo sostituiva - alla individuazione delle "differenze essenziali e formali" tra le forme di governo perseguita da Bodin con la sua scienza politica - la ricerca delle differenze ultime che nella realtà storica venivano a distinguere e la monarchia di Francia da quella di Spagna e la repubblica degli svizzeri da quella degli olandesi. Quindi, a riconoscere con precisione le forme dei vari governi non sarebbe stata più sufficiente l'applicazione della tipologia tradizionale che ne operava in prima istanza e in modo aprioristico la differenziazione secondo il numero degli occupanti la soglia di dominio, ma sarebbe stato necessario che la ragion di stato dispiegasse un'analisi empirica, capace di rivelarne i caratteri peculiari.

Che, poi, la ragion di Stato attagliasse la propria pratica alla "cognitione de' particolari", per il vero sarebbe stato approdo ribadito da Scipione Chiaramonti (1565-1652), a chiusa, per così dire, dell'ampio dibattito che era stato aperto nel 1589 dal capolavoro di Giovanni Botero. Così, Chiaramonti nella sua Della Ragion di Stato, pubblicata nel 1635, tornando a sottolineare Come la politica, e Ragione di Stato siano tra loro differenti, veniva con forza a ribadire il carattere eminentemente empirico che la ragion di stato veniva a testimoniare nel suo esercizio:

${ }^{63}$ G. Costa, Ragionamento sopra la triegua dei Paesi bassi conchiusa in Anversa l'anno MDCIX, In Genova, appresso Giuseppe Pavoni, 1610, p. 34. Nato a metà del Cinquecento, dopo il 1629 non si registrano più notizie né in merito alla sua attività di storiografo né in merito alla sua azione all intemo delle magistrature cittadine, cfr. G. NUTI, COSTA GIOVANNI, in DBI, vol. XXX, 1984, pp,186-188. 
"Quelli che dicono la ragion di Stato essere parte della politica, cioè della cognitione, cioè della cognitione, o facoltà politica scritta ne' libri politici non dicono bene; perché prima la ragion di Stato buona abbraccia la cognitione de' particolari, e le facoltà della buona consultatione, $[. .$.$] le quali non$ si includano nella scientia morale, potendo esserne uno ben dotto, e mancar di queste parti"64.

\begin{abstract}
In the second half of the XIX ${ }^{\text {th }}$ century and during the whole $\mathrm{XXT}^{\text {th }}$ century, the analysis of the different forms of government was considered a fundamental step leading to the foundation of the political theory, even though the complexity of this research was clearly recognized. The difficulty lay in the fact that the scholars' analysis could not catch the great variety of the political systems established through history. This essay shows that such difficulties were already been highlighted by some Italian political thinkers during the Counter-Reformation. In particular, the writers interested in reason of state criticized the schematism of the various forms of government adopted by Aristotle in his Poli-
\end{abstract}

tics and by Jean Bodin in his Six livres de la République (1576). Among these authors, Lodovico Zuccolo is no doubt one of the leading figures. He wrote Considerationi Politiche e Morali (1621) in which he used the reason of state and the "ultimate differences", showing how this technique could detect among the various forms of government to learn "not only how monarchy differs from tyranny" but more precisely how "the French Monarchy differs from the Spanish one or the Swiss popular government differs from the Dutch one", hereby underlining the necessity for the analysis of the forms of government to adopt a more empirical approach.

${ }^{64}$ S. Chiaramonti, Della Ragion di Stato [...]. Nel qual Trattato da primi principij dedotto si scuoprono appieno la natura, le massime, e le specie de' Governi buoni, e de' cattivi, e mascherati, In Fiorenza, Nella Stamperia di Pietro Nesti, 1635, p. 481. Sulla figura di Chiaramonti, filosofo e matematico, si veda l'importante contributo di G. BENZONI, Chiaramonti, Scipione, in DBI, vol. XXIV, 1980, pp. 541-549. 